\title{
New developments in the ROOT fitting classes
}

\author{
Xavier Valls ${ }^{1,2, *}$, Lorenzo Moneta ${ }^{1}$, Guilherme Amadio ${ }^{1}$, and Arthur Tsang ${ }^{3}$ \\ ${ }^{1}$ CERN, Geneva, Switzerland. \\ ${ }^{2}$ Universitat Jaume I, Castelló de la Plana, C. Valenciana, Spain. \\ ${ }^{3}$ Stanford University, Stanford, California, United States of America.
}

\begin{abstract}
.
The ROOT Mathematical and Statistical libraries have been recently improved both to increase their performance and to facilitate the modelling of parametric functions that can be used for performing maximum likelihood fits to data sets to estimate parameters and their uncertainties.

First, we report on the new functionalities introduced in ROOT's TFormula and TF1 classes to build these models in a convenient way for the users. We show how function objects, represented in ROOT by TF1 classes, can be used as probability density functions and how they can be combined together-via an addition operator - to perform extended likelihood fit of several normalized components. We also describe the new operators introduced to perform the convolution of two functions.

Finally, we report on the improvements in the performance of the ROOT fitting algorithm, by using SIMD vectorization when evaluating the model function on large data sets and by exploiting multi-thread parallelization when computing the likelihood function.
\end{abstract}

\section{Introduction: Fitting and modeling in ROOT}

Fitting of data distributions, a fundamental operation in High Energy Physics (HEP) analysis, is one of the most demanding and computing-intensive activities when performing data analysis on the results of the LHC.

ROOT [1] offers several techniques for fitting and modeling data distributions, from integration of the most popular minimizers, to objective function classes, to function classes for the user's provided model function to interface with the minimizer. In this paper, we introduce the recent improvements implemented in the mathematical libraries that make fitting directly in ROOT easier and faster.

We extend the functionality of the TFormula class in ROOT, adding argument parsing and allowing to freely pass variables and parameters into pre-defined and user-defined functions. We also define a new syntax to use certain function compositions techniques, namely normalized sums and convolutions, directly in the TF1 function class. Finally, we introduce parallelization at data-level in the mathematical libraries and parallelization both at task-level and at data-level in the objective functions of the fitting, testing the potential to speed up parallelizable computations.

\footnotetext{
*e-mail: xavier.valls.pla@cern.ch
} 


\section{Modeling the fit with TFormula and TF1}

ROOT's fitting interfaces mainly rely on two classes to handle mathematical functions, TFormula and TF1. We introduce new developments in both classes, such as improved support and argument expression of TFormula function definitions and the introduction of new operators in TF1 to express two methods of function composition: the normalized sum of functions and the convolution.

\subsection{Extended support and improved argument expression in TFormula}

TFormula provides the user with a convenient way to express mathematical functions as strings, e.g. "x+y[0]*59". These expressions are Just in time (JIT) compiled into C++ code.

Recently, we extended TFormula's features with:

- Ordered and named parameters: we can specify which parameter to use from the parameter list by specifying its position. We can also name parameters to improve the readability of the formula (Listing 1, lines 1-4).

- Improved support for multi-dimensional functions: we added support to build TF2 and TF3 from TFormula expressions (Listing 1, lines 5-6 ).

- Concatenation of formula expressions: now it is possible to reuse previous declared functions in a new formula (Listing 1, lines 7-9).

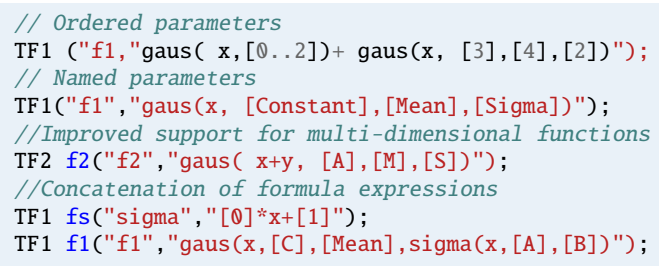

Listing 1: Examples of new ways of expressing parameters in TFormula.

\subsection{New operators: Normalized Additions and Convolutions}

Many typical HEP fits can be formulated as the sum of functions modeling different processes as separate components (e.g. signal + background component). Fitting is often used to determine the fractions or number of events involved in each one of these processes, in order to obtain relevant quantities and indicators, e.g. from the number of events we can obtain cross-sections and discovery significances.

When fitting for number of events, it is usually more interesting to normalize the different model functions before fitting instead of integrating them afterwards, which complicates the estimation of uncertainties due to correlations. A normalized sum is a weighted sum of functions, $f_{i}$, where the coefficients, $c_{i}$, are computed after normalizing each function:

$$
N S U M(f 1, \ldots, f n)(x, p)=\sum_{i=1}^{n} c_{i} \cdot \frac{f_{i}(x, p)}{f_{i}(\xi, p) d \xi},
$$

where NSUM is a numerical aproximation to the expression on the right. 
If each $f_{i}$ gives a distribution of events from a given process $i$, then normalization has the advantage that $c_{i}$ now represents the count of these events.

TF1 is the ROOT class representing a one dimensional parametric function defined between lower and upper limits. It can be constructed from a $\mathrm{C}++$ free function pointer, a TFormula-like expression or using a $\mathrm{C}++$ function or callable object, e.g. a lambda or a functor. In order to simplify performing normalized sum fits, we introduced in TF1 the new operator "NSUM", employed to create composite TF1 function objects from formula-based functions. Listing 2 showcases how to perform a fit with normalized sums.

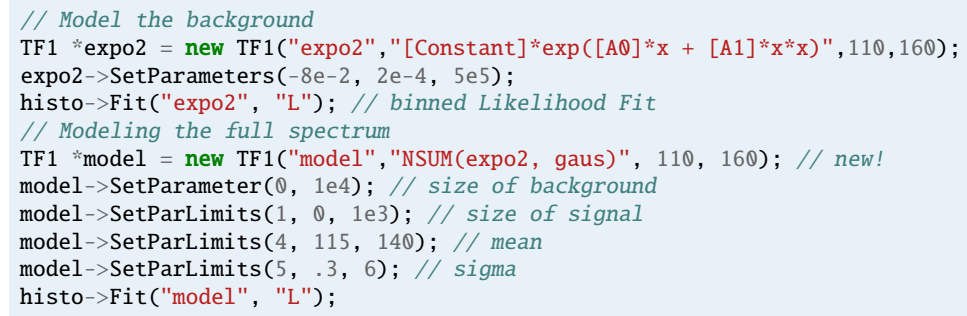

Listing 2: Gaussian signal plus exponential background fit. We define first the background as a double exponential and then we model the full spectrum summing it with a Gaussian representing the signal.

The convolution of $f$ and $g$, is defined as:

$$
(f * g)(x)=\int_{-\inf }^{\mathrm{inf}} f(\xi) g(x-\xi) d \xi
$$

During HEP analysis, we often need to perform the convolution of two functions, e.g. when we compare against a model where $f$ provides a theoretical distribution and $g$ a resolution function. We introduced the new "CONV" operator for building a TF1 function from the convolution of two functions. This operator is translated under the hood to the TF1Convolution class.

Listing 3 presents an example of the use of this operator to perform the convolution of a Breit-Wigner function and a Gaussian function. The convolution can be performed as an fast Fourier transform convolution (by default) or by applying numerical integration.

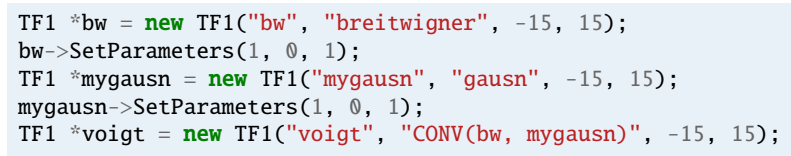

Listing 3: Code necessary for building a TF1 convolution of the Breit-Winger and Gaussian functions.

\section{Multi-level parallelism in ROOT's mathematical classes}

In order to tackle the computational challenges posed by the ambitious physics program of the LHC, ROOT is undergoing a significant update, adapting its codebase for the efficient exploitation of current and future hardware resources, where parallelism plays a central role.

In the context of this extensive modernization effort, we provided data-level parallelization in the mathematical libraries and added support for both task-level and data-level parallelism in the fitting classes. 


\subsection{Data-level parallelism}

Data-level parallelism is achieved in ROOT by exploiting SIMD array operations (vectorization). For this, we rely in the VecCore [2] vectorization library, which we integrated in ROOT's mathematical libraries.

VecCore provides efficient vectorization by offering an extra layer of abstraction on top of other vectorization libraries such as Vc and UME::SIMD. This extra layer of abstraction allows us to write portable, architecture-oblivious code, that will map to the system characteristics on compilation.

VecCore performance has been analyzed by computing, for different VecCore backends and $\mathrm{C}++$ compilers, the Julia Set $z^{2}+0.7885 e^{i \alpha}, \alpha \in(0,2 \pi]$ [3]. The results displayed in Figure 1 shows how Vc outperforms UME::SIMD every time and that gcc is the best compiler choice regarding performance when compiling with AVX2 as the instruction set.

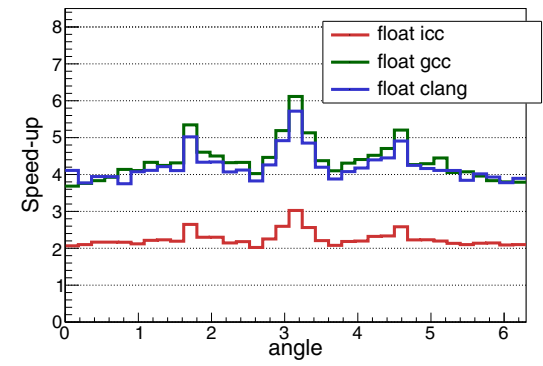

(a)

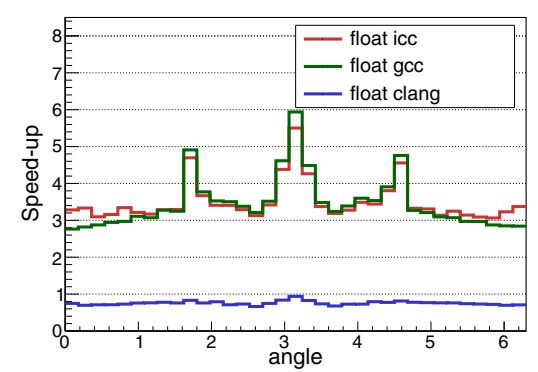

(c)

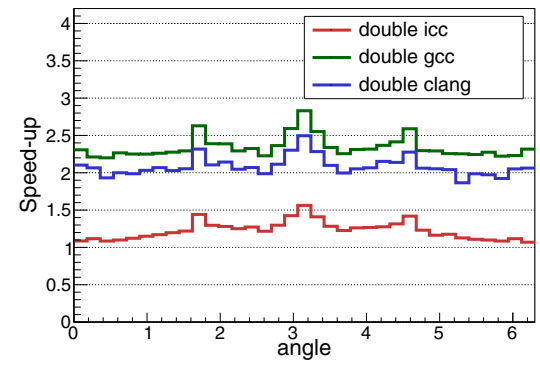

(b)

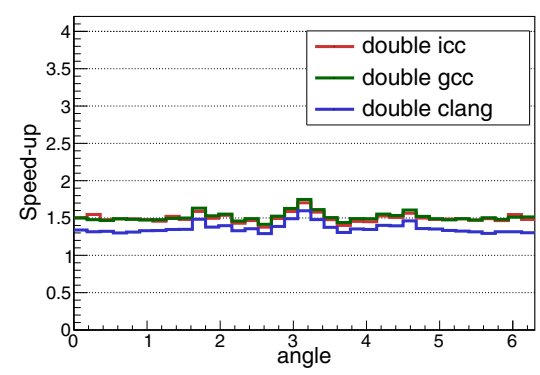

(d)

Figure 1: Performance of the different compilers (clang 5, gcc 7 and icc 17), when evaluating the Julia Set $z^{2}+0.7885 e^{i \alpha}$ with the angle $\alpha \in(0,2 \pi]$ on arrays of float and double SIMD types for VecCore's Vc (1a and 1b) and UME::SIMD (1c and 1d) backends.

We applied vectorization in several places in ROOT, fundamentally in the mathematical functions. For user convenience, we introduced two new ROOT SIMD types, R0OT: :Double_v and R0OT: :Float_V. TF1 now accepts user functions built with ROOT : : Double_v data and return types, and TFormula data types can be compiled to ROOT SIMD types by calling TFormula: : SetVectorized() or TF 1: : SetVectorized(). 
Figure 2 compares, for the evaluation of two different operations, the difference in performance of a free $\mathrm{C}++$ function vectorized, a TF1 built from a vectorized free function and a TF1 built from a formula and then set to vectorized.

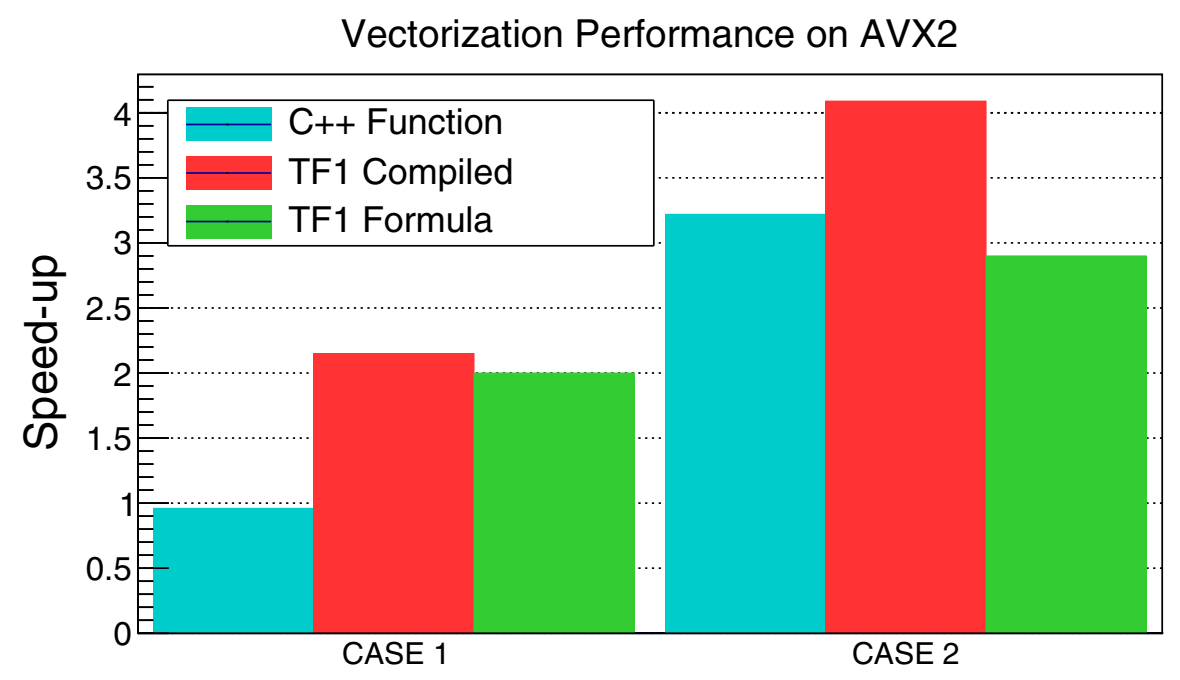

Figure 2: Performance of the vectorization of a free function, a TF1 and a TF1 built from a formula for the evaluation of two different operations, the evaluation of a 2 nd degree polynomial (CASE1) and the sum of and exponential and a Gaussian function (CASE 2).

\subsection{Task-level parallelism}

We exploit task-level parallelism in ROOT with the executors [4], which are highly-reusable implementations of the Map-Reduce pattern recently introduced in ROOT that offer a flexible and convenient programming model and remarkable performance.

ROOT offers several executors that have been deployed successfully throughout ROOTs codebase:

- TSequentialExecutor, a sequential implementation of the MapReduce pattern.

- TProcessExecutor, a fork-based multiprocess executor.

- TThreadExecutor, a TBB-based multithreaded one.

- TNUMAExecutor, a proof of concept aiming at reducing the negative impact of remote memory accesses in NUMA systems by integrating TProcessExecutor, TThreadExecutor and libnuma [3].

\subsection{The fitting case}

VecCore and the executors are applied together in order to achieve the multi-level parallelization of the fitting process [5].

Listing 4 exposes the code needed to perform a fit parallelized both at data-level and at task-level. Note that the code written is the same as in the sequential case except for line 2 , where we activate the implicitly parallel mode of ROOT [4] and line 8, where we set 
the formula expression to be compiled with R0OT: :Double_v data types instead of double types.

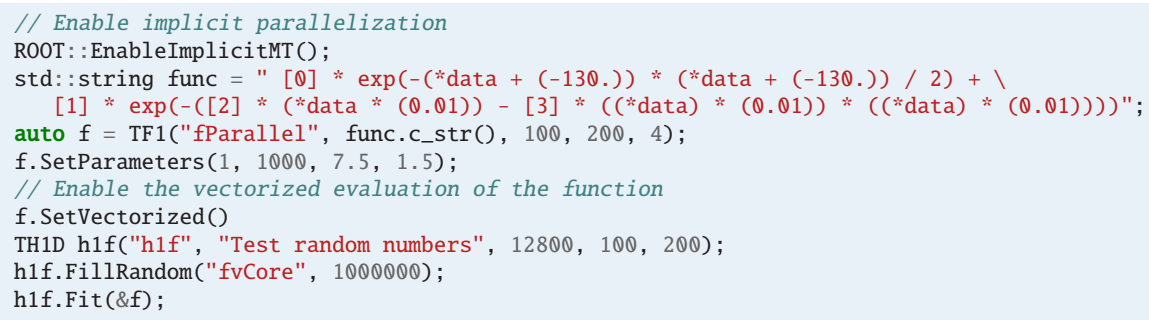

Listing 4: Vectorized and parallelized fit, built from a ROOT formula, of the invariant mass distribution for the diphoton system resulting from the decay of a Higgs boson.

\section{Parallelization of the fitting process}

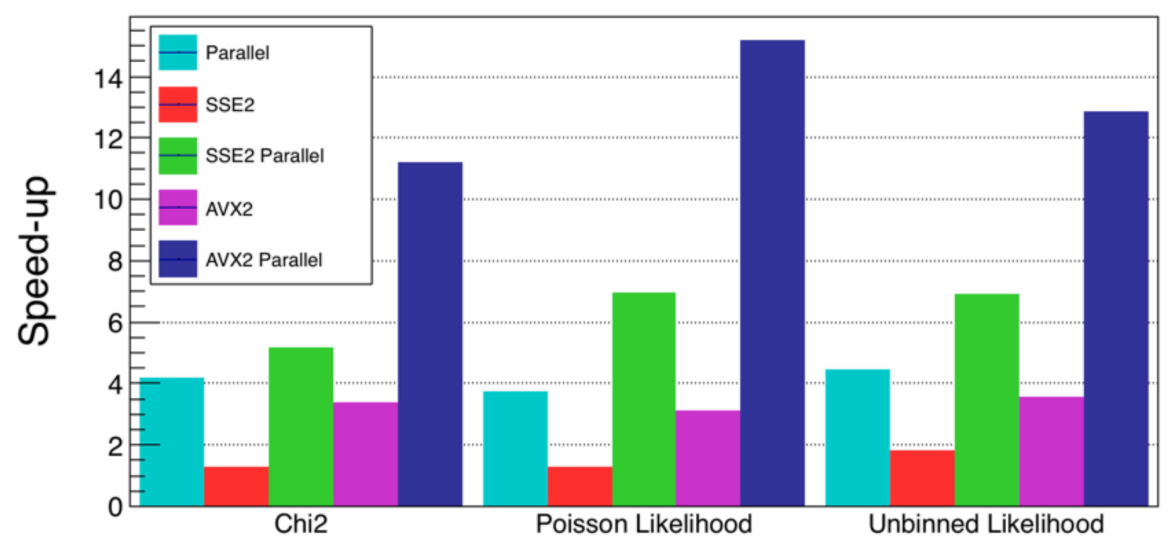

Figure 3: Performance of the multithreaded and vectorized parallelization of the fit with different objective functions and different set of instructions (SSE, or Streaming SIMD Extensions, and AVX2 or Advanced Vector Extensions 2, in an Intel Core i7-4790 processor with 4 physical cores and 8GB of RAM.

Figure 3 presents the performance of evaluating each type of parallelized objective function when fitting for the invariant mass spectrum to determine significance and location of the signal (e.g. $H->\gamma \gamma$ ). The results obtained are good when vectorizing and excellent when parallelizing on several cores, obtaining the most outstanding results when applying simultaneously data-level and task-level parallelization.

\section{Conclusions}

In the context of ROOT's modernization effort, the mathematical and statistical libraries are being improved, extending their functionalities and features and adapting their code for parallelism, capitalizing on the performance the current architectures have to offer.

TFormula and TF1 are at the core of ROOT's mathematical functionalities. We enhanced TFormula's parameter expression, allowing position-aware and named parameters. In addition, we added support for TFormula constructors in multidimensional function classes (TF2, 
TF3). TF1's formula constructor has been extended with the addition of two new operators to generate composite functions, NSUM and CONV, implementing, respectively, the normalized sum and the convolution of two functions.

We introduced a new library in ROOT, VecCore, to implement data-level parallelism in ROOT. VecCore allows for the right level of abstraction from compiler intrinsics and provides a convenient programming model for the user. We analyzed VecCore's performance with different combinations of data types, backends and compilers, concluding that gcc 7 outperforms clang 5 and icc 17, and suggesting to choose Vc over UME::SIMD.

Finally, we demonstrated that the fitting is a perfect candidate for applying parallelization at multiple levels, by exploiting VecCore and the new parallel MapReduce classes-the executors, obtaining remarkable speed-ups when vectorizing and close to ideal when parallelizing the fit over multiple cores, resulting in an increase in performance severalfold without impacting the programming model.

\section{References}

[1] R. Brun, F. Rademakers, ROOT - An Object Oriented Data Analysis Framework, in Proceedings AIHENP'96 Workshop, edited by N. Inst., M. in Phys. (1997), Vol. A389, pp. 81-86, http: //root. cern

[2] G. Amadio, P. Canal, S. Wenzel, root-project/veccore: Release v0.4.2 (2017), https: //github.com/root-project/veccore/

[3] X. Valls Pla, Ph.D. thesis, Universitat Jaume I (2018)

[4] P. Canal, G. Ganis, E. Guiraud, P. Mato Vila, L. Moneta, D. Piparo, E. Tejedor, X. Valls $\mathrm{Pla}$, Expressing Parallelism in ROOT, in 22nd International Conference on Computing in High Energy and Nuclear Physics (IOP, in press)

[5] X.V. Pla, L. Moneta, Parallelization and vectorization of ROOT fitting classes, in Journal of Physics: Conference Series (IOP Publishing, 2018), Vol. 1085, p. 032024 